\title{
La domesticación de Stenocereus stellatus (Pfeiffer)
}

\section{Riccobono (CActaceae)}

\author{
Alejandro Casas ${ }^{1}$, Alfonso Valiente-Banuet ${ }^{2}$ y Javier Caballero ${ }^{3}$ \\ 'Instituto de Ecología, Departamento de Ecología de los Recursos Naturales, Universidad Nacional Autónoma de \\ México. Apdo. Postal 27-3 (Xangari), Morelia, Michoacán, 58089. \\ ${ }^{2}$ Instituto de Ecología, Departamento de Ecología Funcional, Universidad Nacional Autónoma de México. Apdo. Postal \\ 70-275 México, D.F. 04510 \\ ${ }_{3}^{3}$ Jardín Botánico, Instituto de Biología, Universidad Nacional Autónoma de México. Apdo. Postal 70-614 México, D.F. 04510
}

\begin{abstract}
Resumen. El propósito general de este estudio es analizar procesos de domesticación mediante formas de manejo silvícola. Se analizan los efectos de la selección artificial sobre la morfología y biología reproductiva de Stenocereus stellatus comparando poblaciones silvestres; silvestres manejadas in situ (manejo silvícola) y cultivadas del Valle de Tehuacán y La Mixteca Baja. La gente selecciona los fenotipos de esta cactácea columnar con frutos relativamente más grandes y dulces, con cáscara delgada y pocas espinas, favoreciendo su abundancia en poblaciones silvestres manejadas in situ y en huertas. En las poblaciones silvestres manejadas in situ prevalecen los fenotipos preferidos por la gente, lo que indica que la selección artificial ha tenido efectos significativos bajo esta forma de manejo. Los fenotipos preferidos son especialmente abundantes en las huertas, lo que sugiere que bajo el cultivo la selección artificial es aún más intensa. En las poblaciones silvestres no manejadas estos fenotipos son escasos o ausentes. Tal divergencia morfológica se mantiene no obstante que el sistema reproductivo de $S$. stellatus es autoincompatible, en todas las poblaciones, y que no existen barreras espaciales ni temporales para el flujo de polen entre poblaciones silvestres y cultivadas. La abundancia de fenotipos favorables en áreas manejadas por la gente puede explicarse como resultado de la selección artificial, mientras que su escasez o ausencia en poblaciones silvestres parece deberse a incompatibilidad de polen entre algunos fenotipos cultivados y silvestres, y podría deberse también a presiones de selección na-
\end{abstract} tural contra algunos fenotipos cultivados.

Palabras clave: biología reproductiva, cactáceas columnares, domesticación, etnobotánica, Stenocereus stellatus Abstract. This study aimed at analyzing processes of domestication by silvicultural forms of management. The effects of artificial selection on morphology and reproductive biology of Stenocereus stellatus are analyzed in wild; wild-managed in situ (silvicultural management) and cultivated populations from the Tehuacan Valley and La Mixteca Baja. People select the phenotypes of this columnar cactus species with relatively larger and sweeter fruits with thinner peel and fewer spines, favoring their numbers in wild populations managed in situ as well as in home gardens. Favorable phenotypes prevail in wild populations managed in situ, indicating that artificial selection has had significant effects under this form of management. These phenotypes are especially abundant in home gardens, which suggests that artificial selection is even stronger under cultivation. Favorable phenotypes are scarce or absent in non-manipulated wild populations. Such morphological divergence is maintained despite the reproductive system of $S$. stellatus, is self-incompatible in all populations, and even when there were not found spatial and temporal barriers for pollen flow between wild and cultivated populations. Abundance of favorable phenotypes in areas managed by people may be explained by action of artificial selection, while absence of some domesticated phenotypes in wild populations seems to be due to pollen incompatibility between some cultivated phenotypes and wild populations, but it could also be due to pressures of natural selection against cultivated phenotypes.

Key words: columnar cacti, domestication, ethnobotany, reproductive biology, Stenocereus stellatus

os procesos de domesticación en plantas general-

mente se han asociado al cultivo de éstas en ambientes controlados, fuera de sus poblaciones silvestres (véase por ejemplo Harlan, 1992; Zohary y Hopf, 1993). No obstante, diferentes autores han mostra- do que en distintas partes del mundo los seres humanos han practicado formas de manipulación de poblaciones y comunidades vegetales silvestres in situ, y sugerido la posibilidad de que estas formas de manipulación involucren procesos de selección arti- 
ficial (véase Rindos, 1984; Harris y Hillman, 1989; Casas et al., 1997a). Sin embargo, la demostración y evaluación de tales procesos aún no han sido efectuadas.

Diferentes autores han descrito formas de manejo de poblaciones de plantas silvestres para un buen número de especies en México (Alcorn, 1981; Bye, 1993; Caballero, 1994; Casas y Caballero, 1996; Casas et al. 1996; Casas et al., 1997) y, al parecer, estas formas de manejo están muy extendidas en toda Mesoamérica (Casas et al. 1997a). Consecuentemente esta región ofrece, en teoría, la posibilidad de estudiar procesos de domesticación de plantas en relación con tales formas de manejo.

Esta investigación constituye un estudio de caso en el que se pretende analizar si los procesos de domesticación ocurren, y en qué medida lo hacen, bajo formas de manejo de la vegetación silvestre (manejo silvícola). El objeto de estudio es la cactácea columnar Stenocereus stellatus (Pfeiffer) Riccobono, la cual, además de ser cultivada, se encuentra silvestre en zonas áridas y semiáridas del centro de México. Algunas poblaciones silvestres de esta especie se encuentran sujetas a condiciones de manejo silvícola, lo que determina un escenario idóneo para los propósitos generales de este estudio.

S. stellatus es conocida popularmente como "xoconochtle" o "pitaya de agosto". Es una de las cerca de 30 especies de cactáceas columnares mexicanas cuyos frutos son conocidos como "pitayas" (Casas, 1997). Es utilizada como alimento humano, forraje, leña, cerca viva y para consolidar bordos en terrazas (Casas et al., 1997b). Constituye una de las especies de cactáceas productoras de fruta más importantes de México (Pimienta-Barrios y Nobel, 1994; Casas et al., 1997b), y está considerada por la International Society for Horticultural Science entre las 10 especies de cactáceas con mayores potencialidades económicas para México, Centro y Sudamérica (Sedgley y Gardner, 1989).

$S$. stellatus ramifica característicamente desde la base y sus ramas, con 8 a 12 costillas, pueden medir hasta $6 \mathrm{~m}$ de largo. Presenta flores con tubo floral relativamente corto (3 a $4 \mathrm{~cm}$ de largo), de color rojo o rosado, formando coronas en el ápice de las ramas (véase Bravo-Hollis, 1956, 1978). Se distribuye particularmente en el Valle de Tehuacán y la porción sur de la Cuenca del Río Balsas, en los estados de Morelos, Puebla, Guerrero y Oaxaca (figura 1). En estado silvestre puede formar parte de selvas bajas caducifolias y matorrales, entre los 600 y $2000 \mathrm{~m}$ de altitud, en áreas con precipitación de 300 a $800 \mathrm{~mm}$ anuales, tanto en suelos calcáreos como en derivados de areniscas y rocas volcánicas. Bajo cultivo, se le encuentra principalmente en huertas y solares tradicionales (Casas et al., 1997b).

En las cuevas del Valle de Tehuacán exploradas por MacNeish (1967) durante los años 60, Smith (1967) identificó frutos de $S$. stellatus (bajo el sinónimo $L e$ maireocereus stellatus) asociados a restos humanos en estratos de aproximadamente 5500 años de antigüedad, lo que permite tener una idea del tiempo durante el cual las culturas locales han interactuado con esta planta. En la actualidad, S. stellatus es utilizada y manejada por al menos 5 grupos étnicos indígenas (nahuas, mixtecos, popolocas, cuicatecos y tlapanecos), quienes al parecer la han estado domesticando.

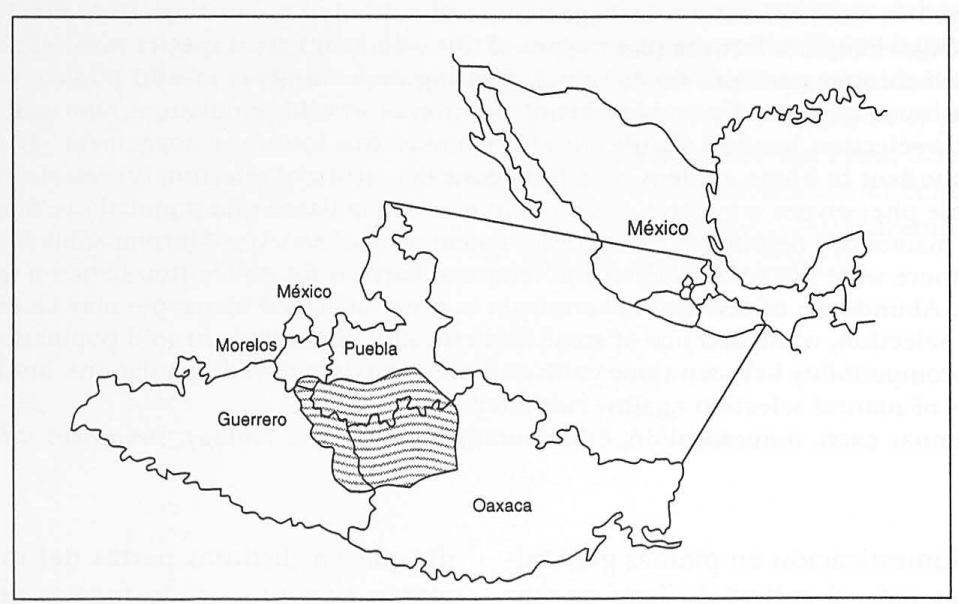

Figura 1. Distribución de Stenocereus stellatus. 
Durante los meses de fructificación (agosto y septiembre), es posible observar una gran diversidad de tipos de frutos de esta especie, en términos de tamaño, color, sabor, cantidad de espinas, y otras características. Ante tal variabilidad, un problema central para analizar el proceso de domesticación de esta especie es saber si dicha variabilidad está determinada únicamente por el amplio espectro de condiciones ambientales en las cuales se distribuye la especie, o si también ha estado influida por selección artificial mediante las diferentes formas de manejo humano que en la actualidad operan sobre esta planta y que podrían haber operado durante cientos o miles de años.

Los objetivos de este estudio fueron evaluar si la selección artificial ha tenido algún efecto sobre los patrones de variabilidad morfológica y la biología reproductiva de $S$. stellatus; analizar las tendencias en la variación de estos aspectos bajo procesos de domesticación, y examinar los mecanismos culturales y biológicos que influyen en el mantenimiento de las diferencias entre poblaciones silvestres, manejadas in situ y cultivadas.

\section{Estrategias de investigación}

Las investigaciones que comprende este estudio se efectuaron en los municipios de Coxcatlán, Tehuacán y Zapotitlán de las Salinas en el Valle de Tehuacán, Puebla y en los municipios de Chazumba, Tequixtepec, Huajuapan de León y Tepexco en la Mixteca Baja, al noroeste de Oaxaca (cuadro 1).

Entre las estrategias generales de investigación se incluyeron estudios etnobotánicos, dirigidos a conocer cuáles son las partes de la planta y los atributos sobre los que se efectúa selección artificial, así como los mecanismos con que opera ésta por parte de la gente que la domestica actualmente. Estos estudios se realizaron en poblados nahuas, popolocas y mixtecos (véase Casas et al., 1997b).

Asimismo, se llevaron a cabo estudios de los patrones de variabilidad morfológica que presenta la especie en diferentes condiciones ambientales (el Valle de Tehuacán y La Mixteca Baja) y bajo distintas formas de manejo humano (recolección en poblaciones silvestres no manejadas; manejo de poblaciones silvestres in situ y cultivo en huertas y solares). El objetivo de estos estudios fue evaluar la hipótesis de que si la selección artificial fuera significativa, sería factible esperar que los patrones de variabilidad morfológica en poblaciones sujetas a manejo difirieran de los patrones presentes en las poblaciones silvestres.

Se midieron y analizaron 23 características de partes vegetativas y reproductivas (cuadro 2 ). Ocho de éstas fueron características que son directamente some-

Cuadro 1. Información general de las poblaciones de Stenocereus stellatus estudiadas. Las extensiones S, M y C en cada población indican su carácter silvestre, manejado in situ y cultivado respectivamente.

\begin{tabular}{|c|c|c|c|c|c|c|c|}
\hline Población & Región & Municipio & $\begin{array}{l}\text { Altitud } \\
\text { (msnm) }\end{array}$ & $\begin{array}{c}\mathrm{TMA}^{1} \\
\left({ }^{\circ} \mathrm{C}\right)\end{array}$ & $\begin{array}{c}\mathrm{PMA}^{2} \\
(\mathrm{~mm})\end{array}$ & Suelos & Hábitat \\
\hline Zapotitlán-S & Tehuacán & Zapotitlán & 1550 & 21.2 & 450 & areniscas & $M X^{3}$ \\
\hline Sn. Juan Raya-S & Tehuacán & Zapotitlán & 1800 & 20.9 & 649.7 & calizas & $M X$ \\
\hline Coxcatlán-S & Tehuacán & Coxcatlán & 1000 & 23.8 & 440.6 & volcanicos & $\mathrm{BTC}^{4}$ \\
\hline Chinango-S & Mixteca & Tequixtepec & 1700 & 20.6 & 720.5 & volcanicos & BTC \\
\hline Tepexco-S & Mixteca & Tepexco & 1150 & 23.0 & 763.7 & volcanicos & BTC \\
\hline Tequixtepec-S & Mixteca & Tequixtepec & 1650 & 20.6 & 720.5 & volcanicos & BTC \\
\hline Metzontla-M & Tehuacán & Zapotitlán & 2000 & 17.2 & 527.9 & limestones & Milpa \\
\hline Sn. Lorenzo-M & Tehuacán & Tehuacán & 1700 & 19.1 & 590.0 & limestones & $M A^{5}$ \\
\hline Coapan-M & Tehuacán & Tehuacán & 1650 & 18.6 & 479.6 & limestones & MA \\
\hline Chinango-M & Mixteca & Tequixtepec & 1700 & 20.6 & 720.5 & volcanicos & Milpa \\
\hline Терехсо-м & Mixteca & Tepexco & 1100 & 23.0 & 763.7 & volcanicos & MA \\
\hline Camotlán-M & Mixteca & Huajuapan & 1600 & 20.6 & 720.5 & volcanicos & MA \\
\hline Huajolotitlán-M & Mixteca & Huajuapan & 1600 & 20.6 & 720.5 & volcanicos & $M A$ \\
\hline Metzontla-C & Tehuacán & Zapotitlán & 1900 & 17.2 & 527.9 & aluvial & Huerta \\
\hline San Lorenzo-C & Tehuacán & Tehuacán & 1700 & 19.1 & 590.0 & calizas & Huerta \\
\hline Zapotitlán-C & Tehuacán & Zapotitlán & 1550 & 21.4 & 450.0 & aluvial & Huerta \\
\hline Chinango-C & Mixteca & Tequixtepec & 1600 & 20.6 & 720.5 & aluvial & Huerta \\
\hline Nochistlán-C & Mixteca & Chazumba & 1650 & 20.6 & 720.5 & aluvial & Huerta \\
\hline Lunatitlán-C & Mixteca & Chazumba & 1650 & 20.6 & 720.5 & aluvial & Huerta \\
\hline
\end{tabular}

${ }^{1}$ Temperatura media anual; ${ }^{2}$ Precipitación media anual; ${ }^{3}$ Matorral xerófito; ${ }^{4}$ Bosque tropical caducifolio; ${ }^{5}$ Milpa abandonada. 
tidas a selección humana. El resto de las características, aunque no son seleccionadas por la gente, se incluyeron en el estudio con el fin de evaluar y comparar patrones de variabilidad morfológica independientemente de la manipulación humana. Se analizaron muestras de 324 individuos de 19 poblaciones tanto del Valle de Tehuacán como de La Mixteca Baja (cuadro 3). Las poblaciones silvestres y manejadas fueron muestreadas por medio de transectos y en las poblaciones cultivadas se muestreó al azar el $10 \%$ de los huertos en seis poblados y el $10 \%$ de los individuos contenidos en éstos, elegidos también aleatoriamente. El análisis de la información se basó en los patrones de clasificación de los individuos dentro de cada región a través de los análisis de componentes principales y discriminante, y en el patrón de clasificación de las poblaciones estudiadas con base en análisis de conglomerados (véase Casas et al., en prensa a).

Se efectuaron además estudios sobre los sistemas de cruzamiento y los mecanismos de polinización de S. stellatus en condiciones silvestres, manejadas in situ y cultivadas, con el fin de analizar si los sistemas reproductivos han sido modificados por la selección artificial, así como para analizar posibles mecanismos de aislamiento reproductivo espacial y temporal entre poblaciones silvestres y cultivadas.

Se efectuaron estudios de biología floral, incluyendo registros temporales de los eventos que ocurren en

Cuadro 2. Características morfológicas analizadas.

\section{Caracteres}

Selección artificial

1. Número de ramas

2. Longitud de la rama mayor

3. Diámetro de la rama mayor

4. Número de costillas

5. Ancho de costillas

6. Profundidad de costillas

7. Número de espinas por areola

8. Tamaño de espina principal

9. Distancia entre areolas

10. Forma de frutos

11. Color de la cáscara

12. Color de la pulpa

13. Sabor de la pulpa

14. Número de areolas por fruto

15. Número de areolas por $\mathrm{cm}^{2}$

16. Grosor de la cáscara

17. Tamaño del fruto

18. Proporción de agua en pulpa

19. Proporción de pulpa

20. Número de semillas por fruto

21. Peso promedio por semilla

22. Peso total de semillas por fruto

23. Proporción de semillas en pulpa las flores durante la antesis. Se evaluó la producción de néctar en muestras de 3 flores cada hora en una población silvestre y una cultivada. Se efectuaron experimentos en el campo con el fin de evaluar si ocurre autopolinización y/o polinización cruzada en individuos silvestres y cultivados, así como para evaluar el papel de los visitantes nocturnos y diurnos en la polinización. Se incluyeron 6 tratamientos distintos en botones florales cubiertos con bolsas de exclusión antes de la antesis: 1] autopolinización no manipulada, dejando cubiertos los botones florales con bolsas de exclusión hasta la maduración o aborción de los frutos; 2] autopolinización manual, depositando polen de una flor en su propio estigma por medio de un pincel; 3] polinización cruzada, depositando manualmente polen de una flor en estigmas de flores de individuos distintos; 4] polinización nocturna, dejando libre acceso a la flor a los visitadores nocturnos, entre las 22:00 h (antes de la apertura de la flor) y las 6:30 h (justo antes del amanecer); 5] polinización diurna, excluyendo a los visitadores nocturnos y dejando libre acceso a los visitadores diurnos entre las 7:00 h (al amanecer) y las 10:00 h (al cerrar completamente las flores); 6] polinización natural, marcando botones florales sin cubrirlos con bolsas de exclusión. Cada tratamiento incluyó al menos 10 botones florales en al menos 8 individuos de cada una de tres poblaciones distintas ( 2 silvestres y una cultivada). Se incluyeron además muestras de botones florales en otras 6 poblaciones con el fin de probar la ocurrencia de autopolinización (tratamientos de autopolinización no manipulada y manual). Se identificaron las especies de animales que visitan las flores y se tomaron muestras de polen en sus cuerpos con el fin de examinar su posible papel como polinizadores. Se efectuaron además estudios fenológicos con el fin de comparar la dinámica de la floración en las poblaciones silvestres y cultivadas incluidas en el estudio. Finalmente, se hicieron experimentos de polinización cruzada con el fin de probar la posible ocurrencia de incompatibilidad de polen entre fenotipos cultivados y silvestres. Se utilizaron bolsas de exclusión para controlar el flujo de polen en las flores receptoras. Se utilizó polen de individuos de variantes domesticadas de pulpa blanca, amarilla, morada y roja y se depositó en los estigmas de flores de individuos silvestres (10 flores por tratamiento). Se incluyó un tratamiento testigo con flores de individuos silvestres (véase Casas et al., en prensa b).

\section{Resultados}

Etnobotánica. Se reconoció que, no obstante la diversidad de usos de Stenocereus stellatus, su principal uti- 
lidad es como alimento humano y que, aunque se pueden consumir los tallos y las flores, la principal parte comestible son los frutos (Casas et al., 1997b).

Se identificaron tres formas de interacción entre la gente y S. stellatus (Casas et al., 1997b). La primera ocurre durante la recolección de frutos en poblaciones silvestres, cuando la gente es capaz de reconocer individuos con diferentes atributos y los recolecta diferencialmente de acuerdo con el uso que les destina. Por ejemplo, los individuos de frutos dulces son preferidos para su consumo como fruta fresca, especialmente si se les va a comercializar, pero para preparar mermeladas o aguas frescas la gente prefiere los frutos agrios.

La segunda forma de interacción es el manejo in $s i t u$, a través del cual la gente tolera selectivamente algunos individuos (generalmente los de frutos más grandes y dulces) durante los aclareos de terrenos para cultivo de maíz. Bajo esta forma de manejo la gente suele incluso plantar ramas de los individuos tolerados con el fin de aumentar su número.

La tercera forma de interacción es el cultivo, principalmente en huertas y solares. En este caso, la gente propaga vegetativamente las ramas de fenotipos deseados procedentes de otras huertas o de las poblaciones silvestres o manejadas in situ. Aunque esta es la forma de cultivo más común, en las huertas también es frecuente el establecimiento de plántulas derivadas de semillas. Los campesinos suelen mantener estas plántulas y eliminarlas o dejarlas en pie, dependiendo de las características de los frutos que producen una vez alcanzado el estado reproductivo (después de 5 a 6 años). El reemplazo de individuos en los huertos, ya sean de origen vegetativo o sexual, se lleva a cabo principalmente cuando los campesinos logran conseguir materiales con características más convenientes.

Las diferentes etnias estudiadas reconocen distintas variedades de $S$. stellatus, las cuales son clasificadas y valoradas de acuerdo con 5 características morfológicas de sus frutos: 1) Tamaño, seleccionando los más grandes; 2) sabor, prefiriendo generalmente los más dulces; 3) color de la pulpa, teniendo mayor prestigio los de color diferente al rojo; 4) espinosidad, optando por los que presentan menor densidad de areolas; y 5) grosor de la cáscara, escogiendo generalmente los de cáscara delgada. Con base en estas características y valores selecciona las plantas silvestres que recolecta, las que tolera, las que planta y las que reemplaza en sus huertos (véase Casas et al., 1997b).

Variabilidad morfológica. El análisis de componentes principales clasificó (figura 2) a la mayor parte de los individuos silvestres de ambas regiones en la porción izquierda de la gráfica, mientras que los individuos manejados y cultivados lo hicieron en la porción central y en la parte derecha, respectivamente. Este análisis permitió identificar que las variables que más contribuyeron a explicar la variación fueron el tamaño de los frutos, el grosor de la cáscara, la densidad de espinas en los frutos, el peso de las semillas, y la proporción de pulpa en los frutos. El sabor de la pulpa fue importante sólo en Tehuacán, mientras que el color de la pulpa lo fue únicamente en La Mixteca (cuadro 4).

$\mathrm{El}$ análisis discriminante estuvo dirigido a probar la hipótesis nula de que no hay diferencias significativas entre los tres grupos de poblaciones definidos de acuerdo con la forma de manejo. El análisis permitió rechazar esta hipótesis (cuadro 5), además de que permitió evaluar el traslape entre poblaciones silvestres, manejadas in situ y cultivadas. En el cuadro 6 puede apreciarse que si bien un alto porcentaje de individuos de cada grupo presenta una identidad

Cuadro 3. Número de individuos de Stenocereus stellatus de poblaciones del Valle de Tehuacán y La Mixteca Baja analizados en los estudios de variabilidad morfológica

\begin{tabular}{|c|c|c|}
\hline Población & Estatus & $\begin{array}{c}\text { Número } \\
\text { individuos }\end{array}$ \\
\hline
\end{tabular}

\section{Valle de Tehuacán}

$\begin{array}{lll}\text { Zapotitlán-S } & \text { Silvestre } & 24 \\ \text { San Juan Raya-S } & \text { Silvestre } & 10 \\ \text { Coxcatlán-S } & \text { Silvestre } & 15 \\ \text { Metzontla-M } & \text { Manejada in situ } & 15 \\ \text { San Lorenzo-M } & \text { Manejada in situ } & 20 \\ \text { Coapan-M } & \text { Manejada in situ } & 10 \\ \text { Metzontla-C } & \text { Cultivada } & 30 \\ \text { San Lorenzo-C } & \text { Cultivada } & 15 \\ \text { Zapotitlán-C } & \text { Cultivada } & 10\end{array}$

\section{Mixteca baja}

$\begin{array}{lll}\text { Chinango-S } & \text { Silvestre } & 20 \\ \text { Tepexco-S } & \text { Silvestre } & 10 \\ \text { Tequixtepec-S } & \text { Silvestre } & 10 \\ \text { Chinango-M } & \text { Manejada in situ } & 15 \\ \text { Tepexco-M } & \text { Manejada in situ } & 10 \\ \text { Camotlán-M } & \text { Manejada in situ } & 10 \\ \text { Huajolotitlán-M } & \text { Manejada in situ } & 10 \\ \text { Chinango-C } & \text { Cultivada } & 50 \\ \text { Nochistlán-C } & \text { Cultivada } & 20 \\ \text { Lunatitlán-C } & \text { Cultivada } & 20\end{array}$


definida, algunos individuos de otros grupos resultaron semejantes al núcleo principal de individuos de cada grupo.

Por otro lado, el análisis de conglomerados clasificó a las poblaciones estudiadas en tres grupos principales (figura 3), cuya composición indica que las poblaciones fueron morfológicamente similares de acuerdo con la región de procedencia, pero también de acuerdo con la forma de manejo a la que se encuentran sujetas (véase Casas et al., en prensa a).

Biología reproductiva. Aún cuando las flores pueden permanecer abiertas en algunos casos hasta las 8 de la mañana, la mayor parte de la antesis ocurre durante la noche (iniciándose a las 8 de la noche) durante la cual se produce una esencia desagradable. Las flores se encuentran completamente abiertas a las 12 de la noche y en ese momento se comienza a liberar el polen. No obstante, para ese momento los estigmas están aún enjutos y la máxima turgencia se registra entre las 2 y las 4 de la mañana, para posteriormente perder turgencia y estar completamente enjutos entre las 6 y 8 de la mañana, cuando las flores aún están abiertas. El máximo pico de producción de néctar se encuentra entre la 2 y 4 de la mañana, co- incidiendo notablemente con el período de máxima turgencia de los estigmas (véase Casas et al., en prensa b). Estas observaciones sugieren fuertemente un patrón de biología floral para la polinización nocturna, lo que resulta consistente con los resultados de los experimentos de polinización. Así, como puede verse en el cuadro 7, todos los tratamientos para autopolinización y polinización diurna fallaron en producir frutos, mientras que los tratamientos para polinización cruzada y para polinización nocturna fueron exitosos. La totalidad de las cruzas experimentales entre variantes cultivadas e individuos silvestres fueron infértiles, mientras que el $60 \%$ de las cruzas usadas como testigo fueron exitosas.

Entre los visitadores nocturnos de las flores de $S$. stellatus destacan cuatro especies de murciélagos (Leptonycteris curasoae yerbabuenae Martínez y Villa; $L$. nivalis Saussure; Choeronycteris mexicana Tschudi y Artibeus jamaicensis yucatanicus J.A. Allen); dos especies de esfíngidos (Eumorpha spp.); y dos especies de coleópteros (Carpophylus spp.). La participación de los murciélagos en la polinización de esta especie parece ser la más importante, pues no sólo las flores presentan rasgos claros de quiropterofilia sino que además, los murciélagos cargan abundante polen y efectúan con-

Cuadro 4. Vectores resultantes del Análisis de Componentes Principales ( $C P=$ Componentes Principales).

\begin{tabular}{|c|c|c|c|c|c|c|}
\hline \multirow[b]{2}{*}{ CARÁCTER } & \multicolumn{3}{|c|}{ Tehuacán } & \multicolumn{3}{|c|}{ Mixteca } \\
\hline & CP1 & $\mathrm{CP} 2$ & CP3 & CP1 & CP2 & CP3 \\
\hline Número de ramas & 0.53 & 0.14 & 0.20 & 0.37 & 0.56 & 0.30 \\
\hline Longitud de la rama mayor & 0.23 & 0.19 & -0.24 & 0.30 & 0.66 & 0.22 \\
\hline Diámetro de la rama mayor & 0.71 & 0.46 & 0.16 & 0.34 & -0.48 & -0.45 \\
\hline Número de costillas & 0.11 & 0.15 & 0.56 & -0.30 & -0.26 & 0.29 \\
\hline Ancho de las costillas & 0.66 & 0.47 & -0.03 & 0.33 & 0.16 & -0.47 \\
\hline Profundidad de las costillas & 0.53 & 0.57 & -0.20 & 0.65 & -0.05 & -0.53 \\
\hline Número de espinas por areola & 0.23 & -0.13 & 0.28 & -0.09 & -0.71 & -0.09 \\
\hline Tamaño de la espina principal & 0.56 & 0.02 & -0.51 & 0.09 & 0.23 & 0.18 \\
\hline Distancia entre areolas & 0.20 & 0.15 & -0.70 & 0.33 & 0.04 & -0.24 \\
\hline Forma del fruto & 0.03 & -0.18 & 0.10 & 0.34 & 0.06 & 0.02 \\
\hline Color de la cáscara & 0.29 & -0.33 & 0.40 & 0.60 & 0.14 & 0.18 \\
\hline Color de la pulpa & 0.25 & -0.24 & 0.17 & 0.71 & 0.12 & 0.03 \\
\hline Sabor de la pulpa & 0.64 & -0.14 & 0.24 & 0.21 & 0.00 & 0.56 \\
\hline Número de areolas por fruto & -0.34 & -0.16 & -0.33 & 0.20 & -0.25 & -0.26 \\
\hline Número de areolas por $\mathrm{cm}^{2}$ & -0.83 & 0.14 & -0.11 & -0.63 & 0.18 & -0.48 \\
\hline Grosor de la cáscara & -0.57 & 0.53 & 0.20 & 0.15 & 0.65 & -0.40 \\
\hline Tamaño del fruto & 0.88 & -0.11 & -0.05 & 0.87 & -0.25 & -0.04 \\
\hline$\%$ de agua en la pulpa & 0.52 & -0.65 & -0.23 & 0.52 & 0.22 & 0.27 \\
\hline$\%$ de pulpa en el fruto & 0.72 & -0.50 & -0.24 & 0.24 & -0.71 & 0.36 \\
\hline Número de semillas por fruto & 0.55 & 0.43 & 0.09 & 0.54 & -0.28 & -0.33 \\
\hline Peso promedio de las semillas & 0.81 & -0.01 & 0.10 & 0.65 & 0.19 & 0.20 \\
\hline Peso total de las semillas & 0.84 & 0.25 & 0.07 & 0.75 & -0.12 & -0.21 \\
\hline$\%$ de semillas en la pulpa & -0.57 & 0.04 & 0.05 & -0.36 & 0.39 & -0.57 \\
\hline
\end{tabular}



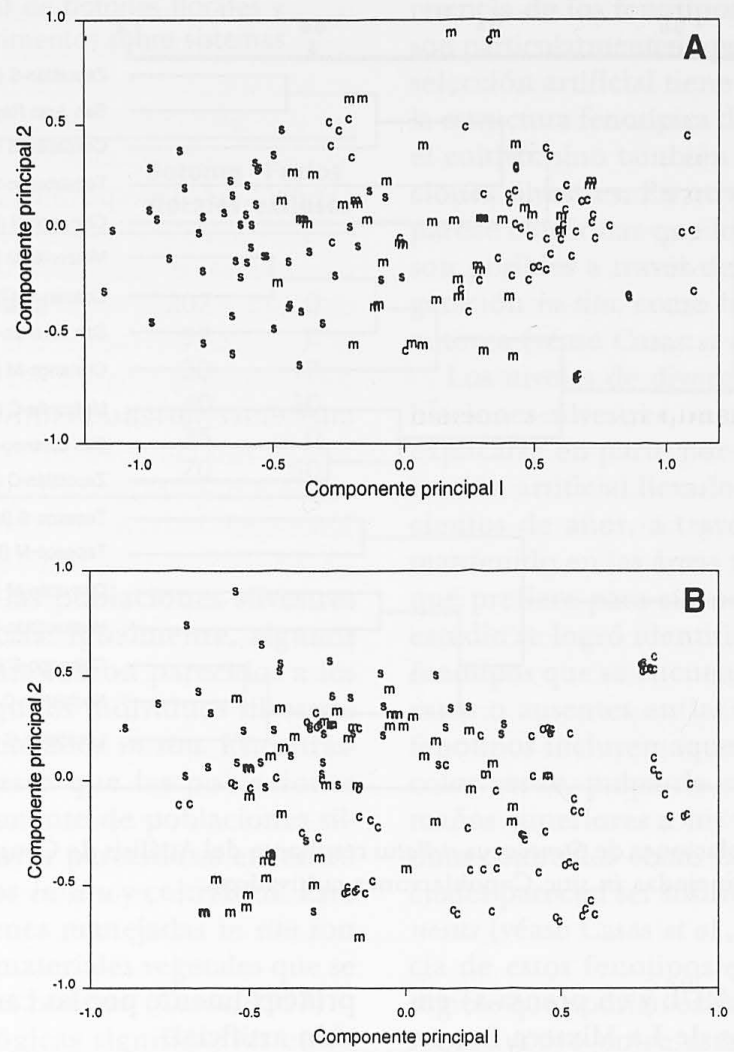

Figura 2. Proyección de individuos de Stenocereus stellatus en el espacio del primer y segundo componentes princi-

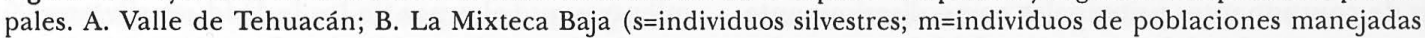
in situ; $\mathrm{c}=$ individuos cultivados).

tacto con los estigmas durante sus visitas a las flores. La participación de los esfíngidos no puede descartarse totalmente pues aunque durante el forrajeo de néctar estos organismos raramente tocan las partes florales internas, se logró identificar polen de $S$. stellatus en muestras de polen tomadas de sus cuerpos. La participación de los coleópteros podría descartarse debido a que al parecer no tocan los estigmas y si, en cambio, en todos los casos fueron observados destruyendo las flores, consumiendo principalmente las estructuras de la base del tubo floral. Además, los coleópteros estuvieron ausentes en todos los tratamientos experimentales de polinización nocturna que produjeron frutos exitosamente.

En la figura 4 puede apreciarse que el período de floración de Stenocereus stellatus fue de entre 90 y 150 días, con pocas flores por individuo produciéndose cada noche y un traspale en la época de producción de flores en todas las poblaciones.

\section{Discusión}

La información etnobotánica indica que: 1] en la actualidad la selección artificial es un proceso real mediante el cual se favorecen algunos fenotipos de Stenocereus stellatus; 2] esta selección se lleva a cabo sobre características de los frutos, las principales partes usadas; 3] los fenotipos preferidos por la gente son aquellos que producen frutos grandes, sabor dulce, pulpa de color diferente al rojo, pocas espinas y cáscara delgada; y 4] la selección artificial se practica tanto bajo la forma de manejo de poblaciones silvestres in situ, como bajo el cultivo en las huertas.

Los análisis de variabilidad morfológica fueron consistentes con esta información. Mostraron que el parecido de los individuos entre sí se relaciona en parte con su procedencia, lo que indica que existen diferencias morfológicas generales entre los individuos del Valle de Tehuacán y los de la Mixteca Baja. En 


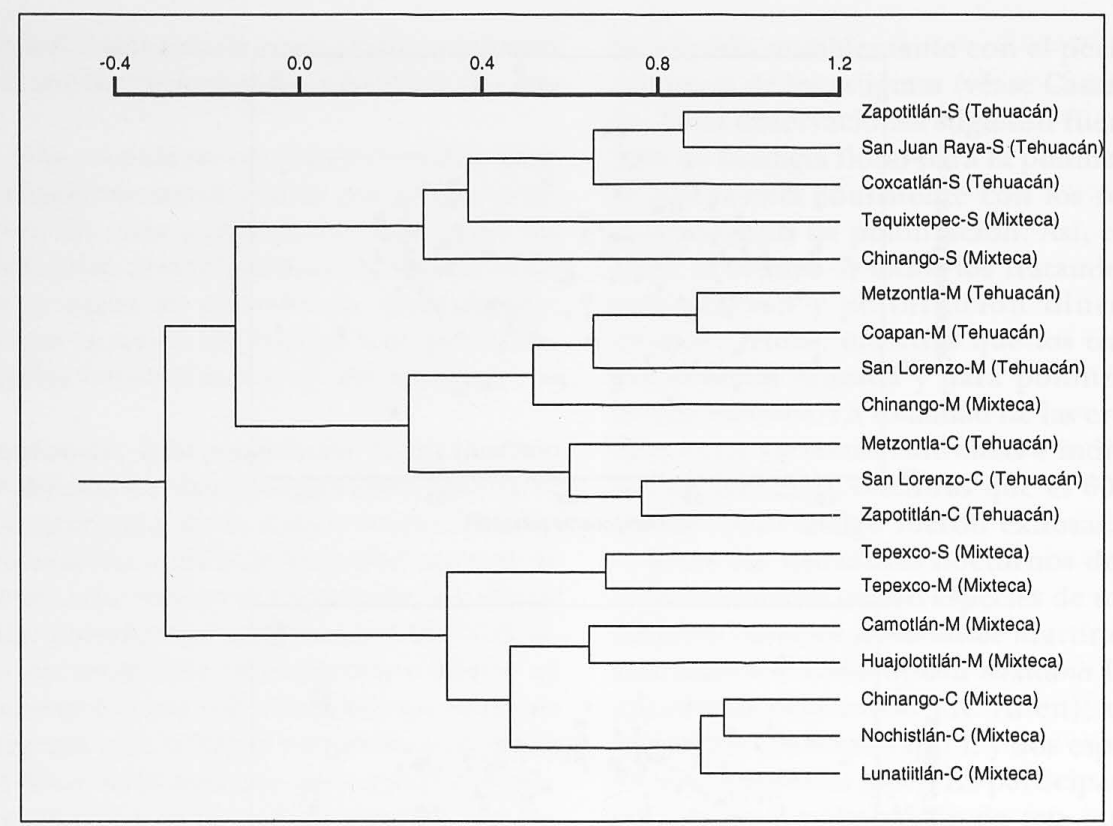

Figura 3. Clasificación de poblaciones de Stenocereus stellatus resultante del Análisis de Conglomerados (S=poblaciones silvestres; $\mathrm{M}=$ poblaciones manejadas in situ; $\mathrm{C}=$ poblaciones cultivados).

otros estudios, Casas et al. (1997b y en prensa a) encontraron que los individuos de La Mixteca fueron más robustos y produjeron frutos y semillas más grandes que los de Tehuacán. No obstante, tanto los análisis de componentes principales como discriminante mostraron que las diferencias morfológicas entre los individuos también se relacionan de manera importante con la forma de manejo de las poblaciones por parte de la gente, y que estas diferencias están dadas principalmente por las características sujetas a selección artificial.

Los estudios morfológicos mostraron que algunos individuos cultivados fueron parecidos a los silvestres, mientras que algunos de los individuos silvestres fueron similares a los cultivados. Lo primero indica que los fenotipos silvestres han mantenido sus características morfológicas aún bajo cultivo, mientras que lo segundo sugiere que los fenotipos cultivados más co-

Cuadro 5. Análisis discriminante para poblaciones silvestres, manejadas in situ y cultivadas de Stenocereus stellatus

\begin{tabular}{|c|c|c|c|c|c|c|c|c|}
\hline \multirow{2}{*}{$\begin{array}{l}\text { Función } \\
\text { discriminante }\end{array}$} & \multicolumn{2}{|c|}{ Eigenvalor } & \multicolumn{2}{|c|}{ Porcentaje relativo } & \multicolumn{4}{|c|}{ Correlación canónica } \\
\hline & Tehuacán & Mixteca & Tehuacán & Mixteca & & acán & & eca \\
\hline 1 & 3.36 & 1.29 & 83.5 & 66.0 & & 88 & & \\
\hline 2 & 0.66 & 0.67 & 16.5 & 34.0 & & 63 & & \\
\hline \multirow{2}{*}{$\begin{array}{l}\text { Funciones } \\
\text { derivadas }\end{array}$} & \multicolumn{2}{|c|}{ Lambda de Wilks } & \multicolumn{2}{|c|}{$X^{2}$} & \multicolumn{2}{|c|}{ Tehuacán } & \multicolumn{2}{|c|}{ Mixteca } \\
\hline & Tehuacán & Mixteca & Tehuacán & Mixteca & GL. & $P$ & GL & $P$ \\
\hline 0 & 0.14 & 0.26 & 275.50 & 221.19 & 38 & 0.00 & 38 & 0.00 \\
\hline 1 & 0.60 & 0.60 & 31.64 & 84.22 & 18 & 0.00 & 18 & 0.00 \\
\hline
\end{tabular}


Cuadro 7. Número total de botones florales y frutos exitosos en experimentos sobre sistemas reproductivos

\section{Tratamiento}

Botones Frutos

florales exitosos

\begin{tabular}{lrr} 
Autopolinización no manipulada & 207 & 0 \\
Autopolinización manual & 98 & 0 \\
Polinización cruzada manual & 30 & 17 \\
Polinización nocturna & 40 & 30 \\
Polinización diurna & 35 & 0 \\
Polinización natural & 70 & 50 \\
\hline
\end{tabular}

munes se encuentran en las poblaciones silvestres aunque en bajas frecuencias. Igualmente, algunos individuos manejados in situ fueron parecidos a los silvestres, mientras que algunos individuos silvestres fueron parecidos a los manejados in situ. Estos traslapes eran esperados puesto que las poblaciones manejadas derivan directamente de poblaciones silvestres. Sin embargo, el mayor parecido se encontró entre individuos manejados in situ y cultivados. Esto se debe a que las poblaciones manejadas in situ son una fuente importante de materiales vegetales que se introducen al cultivo en las huertas (Casas, 1997).

Las diferencias morfológicas significativas entre poblaciones silvestres, manejadas y cultivadas parecen confirmar que los patrones de variabilidad en frutos de S. stellatus han sido influidos por el manejo humano. La divergencia morfológica más marcada se encontró entre las poblaciones silvestres y cultivadas, donde el reemplazo de individuos es relativamente intenso. Pero la divergencia es también importante entre las poblaciones silvestres y manejadas in situ, donde la selección es dirigida a incrementar la fre- cuencia de los fenotipos favorables. Estos resultados son particularmente importantes, pues muestran que la selección artificial tiene un efecto significativo sobre la estructura fenotípica de las poblaciones no sólo bajo el cultivo, sino también bajo el manejo de las poblaciones silvestres. En otras palabras, esta información parece confirmar que los procesos de domesticación son posibles a través de formas de manejo de la vegetación in situ, como ha sido sugerido por diversos autores (véase Casas et al., 1997a).

Los niveles de divergencia morfológica entre poblaciones silvestres, manejadas y cultivadas pueden explicarse en parte por la acción de procesos de selección artificial llevados a cabo probablemente por cientos de años, a través de los cuales la gente ha mantenido en las áreas antropogénicas los fenotipos que prefiere para su uso. No obstante, durante este estudio se logró identificar bajo cultivo un grupo de fenotipos que se encuentran en las huertas y que son raros o ausentes en las poblaciones silvestres. Estos fenotipos incluyen aquellas variantes con cáscara de color verde, pulpa de colores distintos al rojo y tamaños superiores a los $80 \mathrm{~cm}^{3}$, así como los individuos conocidos como "xoconochtle aventurero", los cuales parecen ser híbridos entre $S$. stellatus y $S$. pruinosus (véase Casas et al., 1997b). La escasez o ausencia de estos fenotipos en las poblaciones silvestres sugiere que podrían existir mecanismos de aislamiento reproductivo entre estas variantes y los individuos silvestres, o bien presiones de selección natural contra estos fenotipos, pues de otra manera, éstos deberían encontrarse en las poblaciones silvestres.

Los estudios de biología reproductiva indican que el sistema de reproducción sexual de $S$. stellatus es autoincompatible y que aparentemente no ha sido modificado por la selección artificial. Asimismo, estos estudios revelaron que 4 especies de murciélagos son los vehículos de polen que con mayor probabili-

Cuadro 6. Clasificación de individuos de poblaciones silvestres, manejadas in situ y cultivadas de acuerdo con el Análisis discriminante

\begin{tabular}{|c|c|c|c|c|c|c|c|c|c|c|c|c|c|c|c|}
\hline \multirow{4}{*}{$\begin{array}{l}\text { Grupos } \\
\text { Reales }\end{array}$} & \multicolumn{15}{|c|}{ Grupos Predichos } \\
\hline & \multicolumn{4}{|c|}{ Silvestres } & \multicolumn{4}{|c|}{ Manejados in situ } & \multicolumn{4}{|c|}{ Cultivados } & \multicolumn{3}{|c|}{ Total } \\
\hline & \multicolumn{2}{|c|}{ No. } & \multicolumn{2}{|c|}{$\%$} & \multicolumn{2}{|c|}{ No. } & \multicolumn{2}{|c|}{$\%$} & \multicolumn{2}{|c|}{ No. } & \multicolumn{2}{|c|}{$\%$} & \multicolumn{2}{|c|}{ No. } & $\%$ \\
\hline & Teh. & Mix. & Teh. & Mix & Teh. & Mix. & Teh. & Mix. & Teh. & Mix. & Teh. & Mix. & Teh. & Mix. & Teh. Mix. \\
\hline Silvestres & 47 & 29 & 95.9 & 72.5 & 2 & 5 & 4.1 & 12.5 & 0 & 6 & 0.0 & 15.0 & 49 & 40 & 100.0100 .0 \\
\hline Manejados in situ & 1 & 5 & 2.2 & 11.1 & 40 & 36 & 88.9 & 80.0 & 4 & 4 & 8.9 & 8.9 & 45 & 45 & 100.0100 .0 \\
\hline Cultivados & 2 & 6 & 3.6 & 6.7 & 9 & 7 & 16.4 & 7.8 & 44 & 77 & 80.0 & 85.6 & 55 & 90 & 100.0100 .0 \\
\hline
\end{tabular}


dad efectúan la polinización en esta planta. Sahley et al. (1993) han observado murciélagos del género Leptonycteris moviéndose en una noche entre 30 y 60 $\mathrm{km}$. a partir de las cuevas que habitan, mientras que las distancias entre las poblaciones silvestres y cultivadas estudiadas son del orden de 2 a $10 \mathrm{~km}$, lo cual sugiere que no existen barreras espaciales para el intercambio de polen entre las poblaciones silvestres y las cultivadas. Por otro lado, los estudios fenológicos indican que tampoco existen barreras temporales para limitar el flujo de polen entre tales poblaciones. No obstante, las cruzas experimentales entre variantes cultivadas e individuos silvestres sugieren la existencia de barreras para el flujo de polen debido a incompatibilidad.

\section{Perspectivas}

El estudio aquí presentado constituye un acercamiento al estudio de la domesticación bajo formas de manejo silvícola. Constituye también un primer diagnóstico de la variabilidad de Stenocereus stellatus y de los posibles factores naturales y culturales relacionados con su origen. No obstante, existen nuevos problemas e hipótesis que se derivan de esta primera aproxima- ción y que marcan la perspectiva de las investigaciones por desarrollar en el futuro.

La consolidación de un modelo que explique los procesos de domesticación in situ, requiere desarrollar aún más estudios de caso que permitan considerar el espectro de situaciones en las que pueden presentarse estos procesos, de acuerdo con las formas de manejo, los objetivos de la selección artificial, el ciclo de vida de la planta y su sistema reproductivo, entre otros aspectos. Los distintos ejemplos de formas de manejo in situ descritos en la literatura, y los que se deriven de nuevas investigaciones, constituyen una valiosa materia prima para efectuar estudios de caso similares al presente que permitirían avanzar en la construcción de dicho modelo de domesticación in situ. Este modelo resulta importante no sólo para explicar los procesos actuales de domesticación, sino para entender el proceso de desarrollo tecnológico que en el pasado condujo al origen de la agricultura (Casas et al., 1997a).

En el caso específico de $S$. stellatus, un resultado importante de los estudios efectuados hasta el momento es que características tales como tamaño de frutos, espinosidad y grosor de la cáscara, así como color

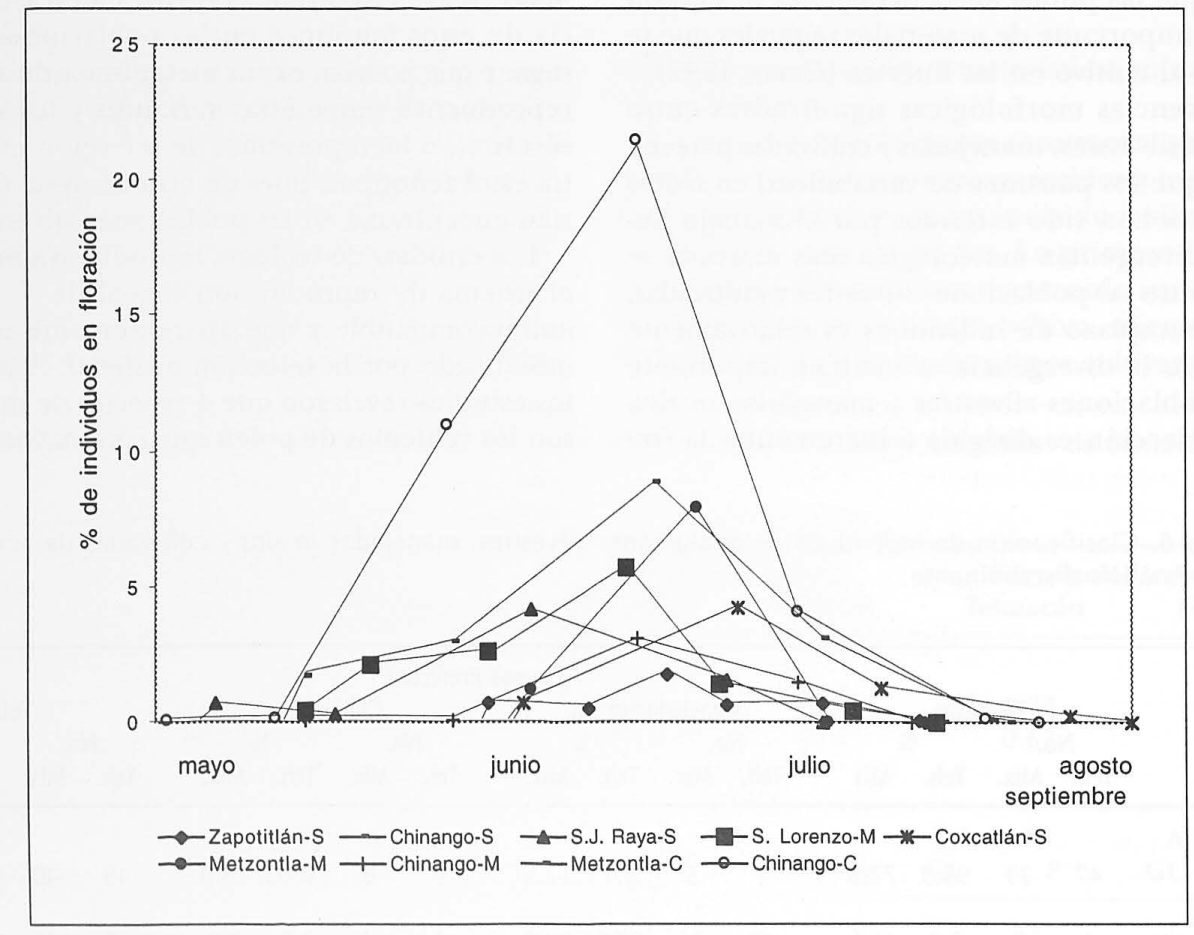

Figura 4. Dinámica de la floración en las poblaciones de Stenocereus stellatus estudiadas. Porcentaje de individuos floreciendo en diferentes momentos de la época de floración. 
de la pulpa, se mantienen constantes en los individuos silvestres introducidos al cultivo en huertas. El hecho de que estas características, además, se expresen diferencialmente en individuos cultivados dentro de un mismo huerto, sugiere que tales características tienen un importante componente genético. Esta información sugiere ya que la selección artificial ha estado influyendo características heredables, determinando procesos evolutivos reales. Sin embargo, para confirmar esta observación, es necesario aún evaluar la expresión de estas características en individuos silvestres y cultivados mantenidos en un ambiente común y mediante experimentos de transplantes recíprocos.

Los experimentos de intercambio de polen entre individuos de variantes cultivadas y silvestres efectuados hasta el momento son aún preliminares. Hace falta aún incluir una muestra más amplia de variantes cultivadas y probar las cruzas de polen de individuos silvestres con gineceos de flores de individuos cultivados. También hace falta aún probar la viabilidad de cruzas entre poblaciones tanto silvestres como manejadas in situ y cultivadas de las dos regiones estudiadas. Al mismo tiempo, resulta importante también realizar experimentos con el fin de probar si la rareza o ausencia de algunos fenotipos cultivados en las poblaciones silvestres se debe a fallas en su dispersión o a la acción de selección natural en contra de la germinación de sus semillas o del establecimiento de sus plántulas.

El estudio de marcadores genéticos tales como polimorfismo de isoenzimas y fragmentos de $\mathrm{ADN}$, puede aportar valiosa información en el estudio del proceso de domesticación de esta especie. En la actualidad, los autores de este trabajo llevan a cabo estudios sobre el polimorfismo en isoenzimas en las mismas poblaciones de individuos analizados desde el punto de vista morfológico. De acuerdo con Gepts (1993), los principales objetivos del estudio de tales marcadores en el análisis de la evolución de plantas cultivadas son, en resumen: 1] identificar los ancestros silvestres de las plantas cultivadas; 2] estudiar la cantidad y organización de variabilidad genética en las especies cultivadas y 3] analizar la introgresión entre plantas silvestres y cultivadas. En el caso específico de $S$. stellatus, el estudio de marcadores genéticos permitiría esclarecer la relación de parentesco entre las poblaciones estudiadas y con ello reconstruir la historia de la domesticación de esta planta, su origen y difusión. Igualmente, permitiría contar con información clave para aclarar el origen y la naturaleza de las variantes exclusivas de las huertas. El estudio de la estructura genética de las poblaciones permitiría analizar el balance entre propagación vegetativa y reproducción sexual bajo diferentes condiciones ambientales en poblaciones silvestres, así como el efecto de la selección artificial en la variabilidad genética. Finalmente, permitiría evaluar el grado de interacción genética entre poblaciones silvestres, manejadas y cultivadas.

Un aspecto central dentro de las perspectivas de estos estudios es el de la conservación y uso de la variabilidad. Del caso de $S$. stellatus, resulta evidente que la materia prima para comprender los procesos de domesticación es la información sobre su variabilidad morfológica y genética, el uso y manejo diferencial que de esta variabilidad han practicado los grupos humanos que han interactuado con esta planta, así como la información sobre biología reproductiva y otros aspectos ecológicos cruciales para entender el estado de variabilidad. Toda esta información permite identificar diferentes líneas fenotípicas con ventajas utilitarias para diferentes actividades productivas que podrían beneficiar los actuales sistemas de producción y abrir programas de fitomejoramiento y biotecnología con múltiples propósitos. El Jardín Botánico de la UNAM alberga ya una colección viva de los individuos que de cada población fueron incluidos en este estudio. Esta colección constituye un embrión de banco de germoplasma para conservación ex situ, el cual podrá alimentarse planificadamente con nuevas colectas. Sin embargo, los programas para la conservación tanto in situ como ex situ de los recursos genéticos contenidos en S. stellatus aún están por diseñarse.

\section{Agradecimientos}

El presente estudio pudo llevarse a cabo gracias al apoyo financiero de la Comisión Nacional para el Conocimiento y Uso de la Biodiversidad (CONABIO), y al apoyo del Instituto de Ecología y Jardín Botánico, Instituto de Biología, UNAM. Agradecemos la valiosa colaboración de la Dra. Patricia Dávila, la Dra. Barbara Pickersgill, José Antonio Soriano, el M. en C. Alberto Rojas y el Dr. Eduardo Morales.

\section{Literatura citada}

Alcorn J. B. 1981. Huatec noncrop resource management: implications for prehispanic rain forest management. Human Ecology 9:395-417.

Bravo-Hollis H. 1956. Iconografía de las cactáceas mexicanas. Stenocereus stellatus (Pfeiffer) Riccobono. Cactáceas y suculentas mexicanas 1:93-95.

Bravo-Hollis H. 1978. Las cactáceas de México. Volúmen I. Universidad Nacional Autónoma de México. México.

Bye R. A. 1993. The role of humans in the diversification of plants in Mexico. En: Ramamoorty, P., Bye R. A., Lot 
A. y Fa J. (eds.) Biological diversity of Mexico. Oxford University Press, New York, 707-731.

Caballero J. 1994. La dimension culturelle de la diversité vegetalle au Mexique. Journal D'Agriculture Traditionalle et de Botanique Apliquee, nouvelle série 36:145-158.

Casas A. 1997. Evolutionary trends in Stenocereus stellatus (Pfeiffer) Riccobono under domestication. Tesis Doctoral. The University of Reading, Inglaterra.

Casas A. y Caballero J. 1996. Traditional management and morphological variation in Leucaena esculenta (Fabaceae: Mimosoideae) in the Mixtec region of Guerrero, Mexico. Economic Botany 50:167-181.

Casas A.; Vázquez M. C., Viveros J. L. y Caballero J. 1996. Plant management among the Nahua and the Mixtec from the Balsas river basin, Mexico: an ethnobotanical approach to the study of domestication. Human Ecology 24:455-478.

Casas A., Caballero J., Mapes C. y Zárate S. 1997a. Manejo de la vegetación, domesticación de plantas y origen de la agricultura en Mesoamérica. Boletín de la Sociedad Botánica de México 61:31-47.

Casas A., Pickersgill B. Caballero J. y Valiente-Banuet A. 1997b. Ethnobotany and domestication in Stenocereus stellatus (Cactaceae), in the Tehuacan Valley and La Mixteca Baja, Mexico. Economic Botany 51:279-292.

Casas A., Caballero J.,Valiente-Banuet A, Soriano J.A. y Dávila P. En prensa a Morphological variation and the process of domestication of Stenocereus stellatus (Cactaceae) in central Mexico. American Journal of Botany.

Casas A., Valiente-Banuet A., Rojas-Martínez A. y Dávila P. En prensa b. Reproductive biology and the process of domestication of the columnar cactus Stenocereus stella- tus (Cactaceae) in central Mexico. American Journal of Botany.

Gepts P. 1993. The use of molecular and biochemical markers in crop evolution studies. En: Hecht M.K. et al. Evolutionary biology. Plenum Press. New York, 51-94.

Harlan J. R. 1992. Origins and processes of domestication. En: Chapman G.P. (ed.) Grass evolution and domestication. Cambridge University Press. Cambridge, 159-175.

Harris D. R. y Hillman G. C. 1989. Foraging and Farming. The Evolution of Plant Exploitation. Unwin Hyman. London.

MacNeish R.S. 1967. A summary of the subsistence. En: Byers D.S. (ed.) The prehistory of the Tehuacán Valley. Volume one: Environment and subsistence. University of Texas Press. Austin, 290-231.

Pimienta-Barrios E. y Nobel P.S. 1994. Pitaya (Stenocereus spp., Cactaceae) an ancient and modern fruit crop of Mexico. Economic Botany 48:76-83.

Rindos D. 1984. The Origins of Agriculture: an Evolutionary Perspective. Academic Press. Orlando.

Sahley C., T. Horner M. A. y Fleming T. H. 1993. Flight speeds and mechanical power outputs of the nectar-feeding bat Leptonycteris curasoae (Phyllostomidae: Glossophaginae). Journal of Mammalogy 74:594-600.

Sedgley M. y Gardner J.A. 1989. International survey of underexploited tropical and subtropical perennials. Acta Horticulturae 250:1-206.

Smith C.E. 1967. Plant remains. En: Byers D.S. (ed.) The prehistory of the Tehuacan Valley. Volume one: Environment and subsistence. University of Texas Press. Austin, 220-225.

Zohary D. y Hopf M. 1993. Domestication of plants in the Old World. Clarendon Press, Oxford. 